\title{
A Chemical Definition of Efficiency
}

\author{
Felix Kaspar ${ }^{1,2 *}$ \\ ${ }^{1}$ Technische Universität Berlin, Institute of Biotechnology, Chair of Bioprocess Engineering, Ackerstraße 76, D- \\ 13355 Berlin, Germany. \\ ${ }^{2}$ BioNukleo GmbH, Ackerstraße 76, D-13355 Berlin, Germany.
}

The term efficient has gained great popularity in the chemical literature, despite the lack of an applicable and relatable definition. In this perspective, a chemical definition of efficiency is discussed building on the concept of non-wasteful resource usage. It is proposed that an efficient method, synthesis or protocol is one which requires less resources in the form of money, time and materials than comparable approaches which accomplish the same task.

Chemistry is, simply spoken, the science of making and analyzing compounds. As such, the synthetic aspect of chemistry is central to many endeavors in today's research, as the ability to synthesize a molecule is a prerequisite to its experimental analysis. Therefore, much of the work at the forefront of current research in the field is focused either on i) making novel compounds, ii) improving methodologies to access known compounds or structural motifs or iii) devising new methods for these purposes. The name of the game here is better, faster, cleaner, easier - i.e. more efficient. Indeed, the use of "efficient" as a descriptor of methods or procedures has become increasingly popular in the literature over the past two decades (Figure 1). In 2019, around 10,000 papers across all major chemistry journals contained the word "efficient". Despite this popularity, a clear definition of the term efficiency in a chemical sense is missing from the literature.

In several dictionaries, ${ }^{[1-3]}$ efficiency is defined as the ability to obtain a product or effect an outcome in a way that does not waste resources, for example in the form of money, time and materials. Conveniently, the relevant metrics to evaluate the efficiency of methods or syntheses in this sense are already at our disposal. Monetary investment can be evaluated via the cost of reagents, time by the number of steps and reaction times and material use through a green chemistry metric of choice. ${ }^{[4]}$ However, the trend in many papers recently is to describe a method as efficient solely based on the yield of a reaction. This ignores other factors such as the origin of the starting materials (i.e. if they had to be synthesized beforehand via a lengthy route of their own) and the amount of reagents, solvents and workup materials used as well as the time it took to perform that synthesis. Clearly, focusing on just one outcome paints an incomplete and potentially misleading picture.

But what exactly does waste mean in this regard? Admittedly, some sort and amount of waste material is a natural byproduct of almost any chemical transformation be it solvents, inorganic salts or undesired reaction products. A similar situation exists for monetary investment and time, as all syntheses require some financial effort and time to perform said transformation. As such, efficiency must be relative, since it requires some form of benchmark to compare the resource investment of a given method to. Herein, the nature of a transformation or target molecule must be considered to avoid a comparison of apples to oranges. Consequently, any method should only be compared to its peers which accomplish the same task. By this logic, methods for an entirely new type of transformation cannot be termed efficient since there is no benchmark to meet.

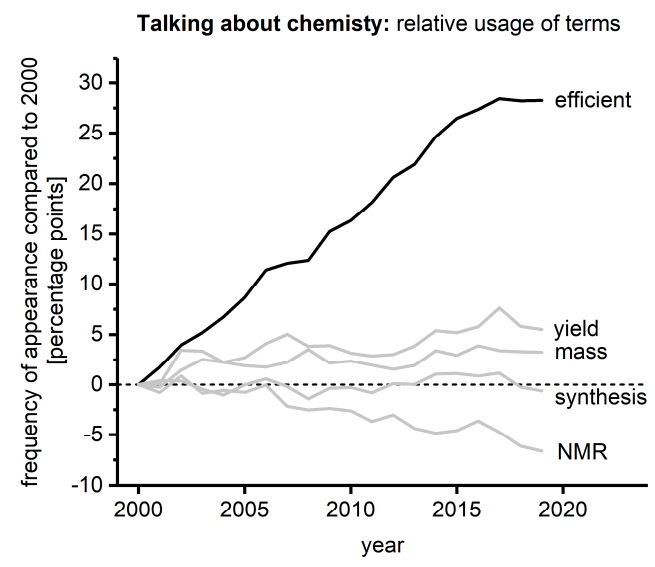

Figure 1. Usage of common terms in articles across leading chemistry journals over the past two decades. This data was obtained from Scopus by searching the archives of 11 chemistry journals for the mentioned keywords. The frequency of papers containing "efficient" increased from $30 \%$ (2000) to $58 \%$ (2019).

\section{Efficiency}

An efficient method, synthesis or protocol is one which requires less resources in the form of money, time and materials than comparable approaches which accomplish the same task.

Money can be evaluated via the cost of reagents.

Time can be assessed through the reaction time as well as the duration of workup and purification procedures.

Material usage can be estimated by a green chemistry metric such as the E-factor or process mass intensity.

The community should certainly be encouraged to continue to strive for more efficient chemistry. However, a more effective use of "efficient" in the literature might be appropriate. Any method which accomplishes a given task is an effective method (i.e. it did the job), but its efficiency should be determined through comparison with other methods for the same task (i.e. how well did it do the job compared to known alternatives?). Thus, to describe a method as efficient one could benchmark this method against alternatives in the literature, for example in terms of number of steps required for the route, cost of reagents or material use. The latter can be evaluated via green chemistry metrics such as the environmental factor (E-factor) ${ }^{[5,6]}$ or process mass intensity (PMI), ${ }^{[7]}$ which are both simple 
mass-based metrics to describe the amount of waste produced for a (hypothetical) kilogram of product. Usually, the E-factor or PMI alone already provide a valuable insight into the overall wastefulness of a reaction or process, since they include all materials used for a transformation or route and correlate with the number of steps. While other green chemistry metrics may also be applied and might provide a comparable picture, consistency in the literature should be advocated and either of the metrics above are recommended for their simplicity and transparency. This, of course, does not mean that methods which are not efficient by the above definition do not have synthetic value (because certainly they do!). Other factors such as an improved substrate spectrum, milder/ambient conditions, simpler/more robust operational procedures or avoidance of hazardous/toxic reagents are all highly relevant and deserve attention from the field.

\section{Acknowledgements}

The author thanks Kerstin Heinecke (Technische Universität Berlin) and M. Rhia L. Stone (Rutgers University) for efficient proofreading and critical comments.

\section{Author Information}

Felix Kaspar, felix.kaspar@web.de, orcid.org/0000-0001-6391-043X

\section{References}

[1] Cambridge Dictionary, "efficiency", https://dictionary.cambridge.org/us/dictionary/engl ish/efficiency, 2020.

" a situation in which a person, company, factory, etc. uses resources such as time, materials, or labour well, without wasting any"

[2] Dictionary, "efficiency", https://www.dictionary.com/browse/efficiency, 2020.

"ability to accomplish a job with a minimum expenditure of time and effort"

[3] Wikipedia, "efficiency", https://en.wikipedia.org/wiki/Efficiency, 2020.

"ability to avoid wasting materials, energy, efforts, money, and time in doing something or in producing a desired result "

[4] R. A. Sheldon, ACS Sustain. Chem. Eng. 2018, 6, 32-48.

[5] R. A. Sheldon, Green Chem. 2017, 19, 18-43.

[6] R. A. Sheldon, Green Chem. 2007, 9, 1273-1283.

[7] C. Jimenez-Gonzalez, C. S. Ponder, Q. B.

Broxterman, J. B. Manley, Org. Process Res. Dev. 2011, 15, 912-917. 